\title{
LOCALLY FLAT IMBEDDINGS OF TOPOLOGICAL MANIFOLDS IN CODIMENSION THREE
}

\author{
BY \\ GLENN P. WELLER( ${ }^{(1)}$
}

\begin{abstract}
This paper presents an imbedding theorem for one topological manifold $M^{n}$ in another topological manifold $Q^{q}$, provided that the codimension $(q-n)$ is at least three. The result holds even if the manifolds are of the recently discovered non-piecewise-linear type. Denote the boundaries of $M$ and $Q$ by $\dot{M}$ and $\dot{Q}$ respectively. Suppose that $M$ is $2 n-q$ connected and $Q$ is $2 n-q+1$ connected. It is then proved that any map $f:(M, \dot{M}) \rightarrow(Q, \dot{Q})$ such that $f \mid \dot{M}$ is a locally flat imbedding is homotopic relative to $\dot{M}$ to a proper locally flat imbedding $g: M \rightarrow Q$. It is also shown that if $M$ is closed and $2 n-q+1$ connected and $Q$ is $2 n-q+2$ connected, then any two homotopic locally flat imbeddings are locally flatly concordant.
\end{abstract}

Introduction. The category of topological manifolds has recently been shown to include manifolds possessing no piecewise linear (P.L.) structure [8]. These non-P.L. manifolds must be investigated in their own right. One approach to the study of topological manifolds is to obtain results analogous to those already known for differentiable and P.L. manifolds.

In the study of imbeddings of manifolds, one of the principal results in the P.L. category is the following:

IRWIN'S IMBEDDING THEOREM [7]. If $n \leqq q-3, M^{n}$ is a compact, $2 n-q$ connected P.L. manifold, $Q^{q}$ is a $2 n-q+1$ connected P.L. manifold and $f:(M, \dot{M}) \rightarrow$ $(Q, \dot{Q})$ is a map such that $f \mid \dot{M}$ is a P.L. imbedding, then $f \sim g$ rel $\dot{M}$ where $g: M \rightarrow Q$ is a proper P.L. imbedding.

This theorem is related to theorems of Haefliger [4] in the differentiable category, although he usually has the dimension requirement that $2 q \geqq 3(n+1)$. This paper presents a proof of the analog of Irwin's theorem for topological manifolds.

The map $g: M \rightarrow Q$ is proved to be a locally flat imbedding. A special case of this theorem was proved by Jack Lees [10] when $\dot{M}=\varnothing$ and $Q$ is euclidean space.

The proof of Irwin's theorem uses the technique of engulfing. Two engulfing theorems for topological manifolds have recently been proved by Newman [13] and Lees [9] and are used extensively here. Furthermore, Miller's P.L. approximation theorem [11] is crucial to this paper.

Received by the editors February 25, 1970.

AMS 1969 subject classifications. Primary 5701, 5705; Secondary 5560, 5570, 5478.

Key words and phrases. Topological manifold, locally flat imbedding, codimension three, concordant imbeddings, engulfing, piecewise-linear manifold.

(1) The author thanks Professor Richard K. Lashof who directed the dissertation of which this paper is a revision. 
Since topological manifolds have P.L. structures locally, P.L. techniques are applied in coordinate neighborhoods. The proof of Theorem 1 then follows by induction on the number of coordinate neighborhoods needed to cover the manifold $M$, with special consideration needed for the last such neighborhood.

1. Some preliminary definitions and lemmas. Let $E^{n}$ denote $n$ dimensional euclidean space and let $H^{n}$ denote the half-space of all points whose last coordinate is nonnegative. An $n$ dimensional topological manifold is a separable Hausdorff space $M^{n}$ in which every point has an open neighborhood homeomorphic to $E^{n}$ or $H^{n}$. If $U$ is an open set in $M$ and $\Psi$ is a homeomorphism of $E^{n}$ or $H^{n}$ onto $U$, the pair $(U, \Psi)$ is called a coordinate neighborhood. The symbol $|\Psi|$ will often be used to denote the image of $\Psi$ when the set $U$ is suppressed. In this case it is understood that $\Psi$ is the relevant homeomorphism.

Let $|\Psi|$ be a coordinate neighborhood where the domain of $\Psi$ is $H^{n}$. The euclidean space $E^{n-1}$ is viewed as a subset of $H^{n}$ in the obvious fashion and the points in $\Psi E^{n-1}$ are called boundary points of $M$. The set of all boundary points of $M$ is an $n-1$ manifold called the boundary of $M$ and denoted $\dot{M}$. Of course, $\dot{M}$ may be empty, but every manifold will be allowed to have a boundary unless the contrary is indicated. The interior of $M$ is $M-\dot{M}$ and is denoted $\dot{M}$.

Let $\alpha>0$. The cube $\left\{x|| x_{i} \mid \leqq \alpha\right\}$ in $E^{n}$ is denoted $C(\alpha)$. If $\Psi: E^{n} \rightarrow|\Psi|$ is a coordinate neighborhood, the symbol $|\Psi|_{\alpha}$ will denote $\Psi C(\alpha)$. Note that $|\Psi|$ is open while $|\Psi|_{\alpha}$ is closed. In the case that $\Psi: H^{n} \rightarrow|\Psi|$, the symbol $|\Psi|_{\alpha}$ will denote $\Psi\left[C(\alpha) \cap H^{n}\right]$.

Any topological manifold $M$ can be imbedded as a closed subset of a sufficiently high dimensional euclidean space [12] and hence has a metric, which will usually be denoted $d$.

Various standard symbols will be used throughout this paper. For instance, $\bar{A}$ will denote the closure of the subspace $A$ in a given space, and " $\sim$ " will mean "is

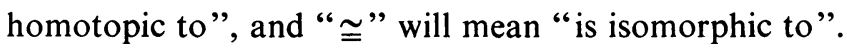

If $Q$ is a manifold with boundary, $\dot{Q}$ has a boundary collar [1]. That is, there is an open set $U$ in $Q, \dot{Q} \subset U$, and there is a homeomorphism $\Psi: \bar{U} \rightarrow \dot{Q} \times[0,1]$ such that if $x \in \dot{Q}, \Psi x=(x, 0)$. Of course, $[0,1]$ may be replaced by any closed interval. The next complicated lemma provides rather nice boundary collars and will be useful in homotoping maps which are imbeddings on the boundary to maps which are imbeddings near the boundary. In the lemma $M_{+}$denotes the manifold obtained from the disjoint union of $M \times 1$ and $\dot{M} \times I$ by gluing along the common subspace $\dot{M} \times 1$. If $M$ is P.L., so is $M_{+}$.

LemMA 1. There is a homotopy $F: M \times I \rightarrow M_{+}$so that

(1) $F_{0}: M \rightarrow M_{+}$is a homeomorphism onto with $F_{0} x=(x, 0)$ for $x \in \dot{M}$.

(2) $F_{t}: M \rightarrow M_{+}$is a homeomorphism into:

(3) $F_{t} x=(x, t)$ for $x \in \dot{M}$. 
(4) $F_{1}(x)=(x, 1)$ for all $x \in M$.

(5) If $V=F_{0}^{-1}(\dot{M} \times[0,1])$, then $F_{0} \mid \bar{V}: \bar{V} \rightarrow \dot{M} \times[0,1]$ is a boundary collar.

There is also a homotopy $G: M_{+} \times I \rightarrow M$ such that

(1') $G_{0}=F_{1}^{-1}$,

(2') $G_{t}(x, s)=x$ if $x \in \dot{M}, s \leqq t$,

(3') $G_{1}(x, 1)=x$ for all $x \in M$,

(4') $G_{1}(x, t)=x$ for all $x \in \dot{M}$.

Furthermore, the boundary collar $\bar{V}$ can be prescribed in advance. If $M$ and $\bar{V}$ are P.L., then $F_{0}$ and $G_{0}$ can be chosen to be P.L.

Proof. Take a boundary collar $\bar{U} \stackrel{\beta}{\rightarrow} \dot{M} \times[1,3]$ where $\beta x=(x, 1)$ if $x \in \dot{M}$. If $\bar{V}$ is given in advance, find a larger collar $\bar{U}$ as above with $\bar{V}=\beta^{-1}(\dot{M} \times[1,2])$. If $\bar{V}$ is a P.L. collar, $\bar{U}$ can be made a P.L. collar [6].

For each $0 \leqq t \leqq 1$ define $h_{t}:[1,3] \rightarrow[t, 3]$ to be the P.L. homeomorphism defined by setting $h_{t}(3)=3, h_{t}(2)=1+t, h_{t}(2-t)=1, h_{t}(1)=t$ and extending linearly. This defines

$$
h:[1,3] \times I \rightarrow[0,3] .
$$

Similarly define $k_{t}:[0,3] \rightarrow[1,3]$ to be the P.L. map obtained by setting $k_{t}(3)=3, k_{t}(1)=2-t, k_{t}(t)=1, k_{t}(0)=1$ and extending linearly. This defines $k:[0,3] \times I \rightarrow[1,3]$. Define

$$
F: M \times I \rightarrow M_{+}, \quad G: M_{+} \times I \rightarrow M
$$

by

$$
\begin{aligned}
F(x, t) & =(x, 1), & & x \notin U, \\
& =\left(\beta^{-1}\left(1 \times h_{t}\right) \beta x, 1\right), & & x \in \beta^{-1}(\dot{M} \times[2-t, 3]), \\
& =\left(1 \times h_{t}\right) \beta x, & & x \in \beta^{-1}(\dot{M} \times[1,2-t]) ; \\
G(x, s, t) & =x, & & x \notin U, \quad s=1, \\
& =\beta^{-1}\left(1 \times k_{t}\right) \beta x, & & x \in \bar{U}, \quad s=1, \\
& =\beta^{-1}\left(x, k_{t}(s)\right), & & x \in \dot{M}, \quad s \geqq t, \\
& =x, & & x \in \dot{M}, \quad s \leqq t .
\end{aligned}
$$

It is routine that all the desired properties hold. Furthermore, if $H_{t}=G_{0} F_{t}$, the proof yields the following:

LEMMA 2. There is an isotopy $H_{t}$ of $M$ into itself such that

(1) $H_{0}=1_{M}$.

(2) $H_{1} M \subset M$.

(3) For some neighborhood $U$ of $\dot{M}, H_{t} \mid M-U=1$.

At this point some preliminary steps toward proving the main result of this paper can be made. But first some definitions must be given. 
Definition 1. Let $M^{n}$ and $Q^{q}$ be topological manifolds and $f: M \rightarrow Q$ be a map. $f$ is said to be proper if $f \dot{M} \subset \dot{Q}$ and $f \dot{M} \subset \dot{Q}$. $f$ is said to be strictly proper if $f$ is proper and there are boundary collars $\varphi: \dot{M} \times[0,1] \rightarrow \bar{U}$ and $\Psi: \dot{Q} \times[0,1] \rightarrow \bar{V}$ such that the following diagram commutes:

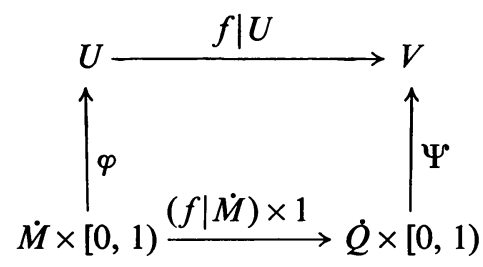

$f$ is said to be locally flat if $f M^{\circ} \subset \dot{Q}$ and for each $x \in M$ there exist neighborhoods $(U, V)$ of $(f x, x)$ in $(Q, M)$ such that $f$ is $1-1$ on $V$ and the pair $(U, f V)$ is homeomorphic to $\left(E^{q}, E^{n}\right)$ if $x \in \stackrel{M}{M}, f x \in \dot{Q} ;$ to $\left(H^{q}, H^{n}\right)$ if $x \in \dot{M}, f x \in \dot{Q}$; or to $\left(E^{q}, H^{n}\right)$ if $x \in \dot{M}, f x \in \dot{Q}$.

The concept of local flatness is related to certain concepts in the differentiable and P.L. categories. If $M$ and $Q$ are differentiable manifolds and $f$ is a differentiable immersion, then $f$ is locally flat by the Implicit Function Theorem [12]. If $M$ and $Q$ are P.L. manifolds and $f$ is a proper P.L. imbedding, then $f$ is called locally unknotted if for every point $x \in M$ there are closed neighborhoods $(U, V)$ of $(f x, x)$ in $(Q, M)$ and a P.L. homeomorphism $h:(U, f V) \rightarrow\left(B^{q}, B^{n}\right)$, where $\left(B^{q}, B^{n}\right)$ is the standard ball-pair. In [16], Zeeman proved that if $q-n \geqq 3$, then $f$ is locally unknotted. Hence, the following lemma is clear.

Lemma 3. If $f$ is a P.L. imbedding of $C(1)$, the cube in $E^{n}$, into $E^{q}$ where $q-n \geqq 3$, then $f \mid \stackrel{C}{C}(1)$ is a locally flat imbedding.

Another useful fact about locally flat maps is stated in the following lemma. In this paper a simplex is always a closed simplex.

LEMMA 4. Let $T$ be a triangulation of $C(1) \subset E^{n}$ with $K$ a subcomplex of $T$. Let $M^{n}$ and $Q^{q}$ be topological manifolds and $f: M \rightarrow Q$ a proper map such that for some open subset $O$ of $\stackrel{M}{M}, f \mid O$ is a locally flat imbedding. Then for any coordinate homeomorphism $\Psi: E^{n} \rightarrow|\Psi| \subset M$ such that $\Psi K \subset O, f \Psi \mid K$ is an imbedding which is locally flat on every simplex of $K$.

Proof. Let $\Delta^{l}$ be a simplex in $K$ and $x \in \Delta$. By hypothesis there are open neighborhoods $\left(W, V^{\prime}\right)$ of $(f \Psi x, \Psi x)$ in $\left(Q^{\circ}, O\right)$ and a homeomorphism

$$
k:\left(W, f V^{\prime}\right) \rightarrow\left(E^{q}, E^{n}\right)
$$


Suppose first that $x \in \stackrel{\circ}{\Delta}$. Then there are open neighborhoods $(V, U)$ of $x$ in $\left(E^{n}, \Delta\right)$ with $U \subset V, \Psi V \subset V^{\prime} \subset O$ and a homeomorphism $h:(V, U) \rightarrow\left(E^{n}, E^{l}\right)$. Then

$$
\begin{aligned}
&\left(E^{q}, E^{n}, E^{l}\right)=\left(E^{n} \times E^{q-n}, E^{n}, E^{l}\right) \stackrel{h^{-1} \times 1}{\longrightarrow}\left(V \times E^{q-n}, V, U\right) \\
& \stackrel{(k f \Psi) \times 1}{\longrightarrow}\left(k f \Psi V \times E^{q-n}, k f \Psi V, k f \Psi U\right) .
\end{aligned}
$$

Since $V$ is open in $E^{n}$ and $k f \Psi: E^{n} \rightarrow E^{n}$ is a homeomorphism, $k f \Psi V$ is open in $E^{n}$ by the Theorem on Invariance of Domain. Hence $k f \Psi V \times E^{q-n}$ is open in $E^{q}$ and $W^{\prime}=k^{-1}\left(k f \Psi V \times E^{q-n}\right)$ is open in $Q$. Thus, there are homeomorphisms $\left(E^{q}, E^{l}\right) \rightarrow\left(k f \Psi V \times E^{q-n}, k f \Psi U\right) \stackrel{k^{-1}}{\longrightarrow}\left(W^{\prime}, f \Psi U\right)$.

If $x \in \dot{\Delta}$, then $h U$ can be chosen to be $H^{l}$ and $\left(E^{q}, H^{l}\right)$ is homeomorphic to $\left(W^{\prime}, f \Psi U\right)$ as before. In either case, $f \Psi \mid \Delta$ is locally flat.

The following two lemmas establish the initial setup for the inductive argument which appears in $\S 3$.

Lemma 5. Let $f:(M, \dot{M}) \rightarrow(Q, \dot{Q})$ be a map such that $f \mid \dot{M}$ is a locally flat imbedding into $\dot{Q}$. Then there is a map $\mathrm{g}: M \rightarrow Q$ such that

(1) with respect to open boundary collars $U$ and $V$ in $M$ and $Q$ respectively, $g$ is strictly proper,

(2) $g \mid U$ is a locally flat imbedding,

(3) $g \sim \operatorname{rel} \dot{M}$.

Furthermore, $U$ may be prescribed in advance provided $\bar{U}$ is a closed boundary collar in $M$.

Proof. Use Lemma 1 twice to obtain $U$ and $F$ for $M$ and $V$ and $G$ for $Q$ with the properties listed in Lemma 1 . Of course, $U$ can be prescribed in advance. Set $g=G_{0}(f \times 1) F_{0}$. Schematically

$$
M \stackrel{F_{0}}{\longrightarrow} M_{+} \stackrel{f \times 1}{\longrightarrow} Q_{+} \stackrel{G_{0}}{\longrightarrow} Q .
$$

Then $g \sim G_{1}(f \times 1) F_{0} \sim G_{1}(f \times 1) F_{1}=f$ and all homotopies are relative to $\dot{M}$.

The remainder of the proof will demonstrate that $g$ is strictly proper and that $g \mid U$ is a locally flat imbedding. Clearly $g \mid U$ is an imbedding. Let $g x=y$ where $x \in U$. Let $F_{0} x=\left(x^{\prime}, t\right), x^{\prime} \in \dot{M}, 0 \leqq t \leqq 1, f x^{\prime}=y^{\prime}$. Then $y=g x=G_{0}(f \times 1) F_{0} x$ $=G_{0}\left(y^{\prime}, t\right)$. Since $f \mid \dot{M}$ is locally flat, there are neighborhoods $U^{\prime}$ and $V^{\prime}$ of $x^{\prime}$ and $y^{\prime}$ in $\dot{M}$ and $\dot{Q}$ respectively and homeomorphisms $h, k$ such that the following diagram commutes, where $i$ is the standard inclusion:

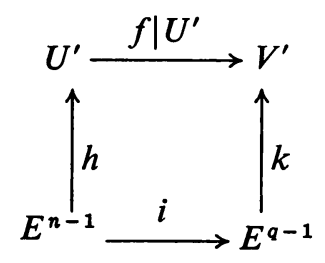


Then the following diagram commutes:

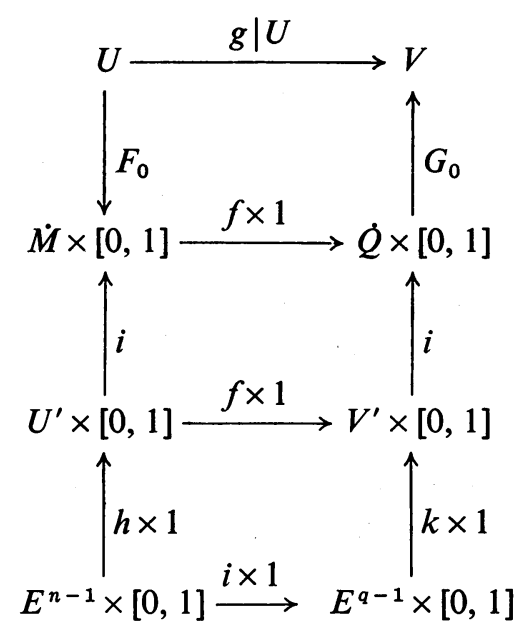

Since $i \times 1$ is obviously locally flat, so is $g \mid U$.

Lemma 6. Let $n \leqq q$ and let $M^{n}$ and $Q^{q}$ be topological manifolds with $\dot{M}=\varnothing$. Let $f: M \rightarrow Q$ be a map and $x_{0} \in M$. Then there is a map $g: M \rightarrow Q$ and an open neighborhood $U$ of $x_{0}$ such that $g \sim f$ and $g \mid U$ is a locally flat imbedding.

Proof. By Lemma 2, after a preliminary homotopy of $f$, it can be assumed that $f M \subset Q$. Let $\varepsilon>0$ be chosen so that any $\varepsilon$-approximation to $f$ is homotopic to $f$. Let $|\varphi|$ be a coordinate neighborhood in $Q$ at $f x_{0}$ of diameter $<\varepsilon$. Let $|\Psi|$ be a coordinate neighborhood at $x_{0}$ such that $f|\bar{\Psi}| \subset|\varphi|$.

Let $i: E^{n} \rightarrow E^{q}$ be the standard inclusion. Let $\mu, \nu$ be a partition of unity on $E^{n}$ subordinate to the open cover $\stackrel{\circ}{C}(2), E^{n}-C(1)$. Let $U=|\dot{\Psi}|_{1}$. Define $F: E^{n} \rightarrow E^{q}$ by $F(x)=\mu(x) i(x)+\nu(x) \varphi^{-1} f \Psi(x)$. Then $F|\dot{C}(1)=i| \dot{C}(1)$ and $F \mid E^{n}-C(2)=\varphi^{-1} f \Psi$. Define $g: M \rightarrow Q$ by

$$
\begin{aligned}
g(x) & =\varphi \Psi^{-1}(x), & & x \in|\Psi|, \\
& =f(x), & & x \notin|\Psi| .
\end{aligned}
$$

Then $g \mid U$ is a locally flat imbedding, $d(f, g)<\varepsilon$, and $f \sim g$.

General Assumption. Throughout the remainder of this paper all manifolds will be assumed to be connected unless otherwise stated.

2. The main geometrical tools. This section contains the main geometrical tools used to prove the principal result of this paper. Particular attention is given to engulfing theorems of Newman and Lees as well as to the approximation theorem of Miller. Specific applications of these important principles are then obtained. All of these tools are essential to the remaining sections of the paper.

Definition 2. A subset $X$ of a topological manifold $M^{n}$ is called locally tame of dimension $p$ if there is a covering of $X$ by open neighborhoods $\left|\Psi_{i}\right|_{1}$ such that $\Psi_{i}^{-1}\left(X \cap\left|\Psi_{i}\right|_{1}\right)$ is a polyhedral set in $E^{n}$ of dimension $p$. 
Newman's Engulfing Theorem [13]. Let $V$ be an open set in a topological manifold $M^{n}$. Suppose that either of the following conditions holds:

(1) $M$ is p-connected, $V$ is $p-1$ connected.

(2) The pair $(M, V)$ is p-connected.

Then every locally tame closed set $X$ of dimension $p \leqq n-3$ such that $X-V$ is compact can be engulfed by $V$. That is, there is a homeomorphism $h: M \rightarrow M$ isotopic to the identity and fixed outside a compact set such that $X \subset h V$.

Lees' Engulfing Theorem [9]. Let $M^{q}$ be a $2 n-q+2$ connected topological manifold without boundary, let $L$ be an $n$-complex, $q-n \geqq 3$ and let $f: L \rightarrow M$ be an imbedding, locally flat on every simplex of $L$.

Then if $f L$ is inessential in $M, f L$ is contained in a coordinate neighborhood.

Outline of Lees' proof. Let $K$ be a triangulation of the cone on $L$ in which $L$ is a subcomplex. Since $f L$ is inessential in $M, f$ extends to a map $f: K \rightarrow M$. Let $K=K_{r} \searrow K_{r-1} \searrow \cdots \searrow K_{0}=$ pt. represent the collapse of the cone where $K$ is chosen so that if $K_{i} \subset K_{i-1} \cup a_{i} A_{i}, a_{i} A_{i} \subset K_{i-1}$, then $f a_{i} A_{i} \subset\left|\Psi_{i}\right|$ for $1 \leqq i \leqq r$. It is important to note that the coordinate neighborhoods $\left|\Psi_{i}\right|$ are indexed as shown but otherwise arbitrarily chosen.

Maps $f_{i}: K \rightarrow M$ are then inductively constructed such that

(1) $f_{i}=f$ on $L$,

(2) $f_{i} a_{j} A_{j} \subset\left|\Psi_{j}\right|, 1 \leqq j \leqq r$,

(3) $f_{i} K_{i}$ is contained in a coordinate neighborhood.

Then $g=f_{r}$ is defined. In particular, $g K \subset|\Psi|$ for some coordinate neighborhood. Hence, $g L=f L \subset|\Psi|$.

Proposition 1. If $n \leqq q-2, D^{n}$ is a P.L. $n$-disk, $Q^{q}$ is a $2 n-q$ connected topological manifold without boundary and $f: D \rightarrow Q$ is a map such that $f \mid \dot{D}$ is an imbedding which is locally flat on every simplex of some triangulation $L$ of $\dot{D}$, then $f \sim g$ rel $\dot{D}$ where $g D$ is contained in a coordinate neighborhood on $Q$.

Proof. Note that $\operatorname{dim} L=n-1, Q$ is $2(n-1)-q+2$ connected and $q-(n-1) \geqq 3$. Extend the triangulation $L$ to a triangulation $K$ of $D$ as the cone on $L$. Now there is an $\varepsilon>0$ so that any $\varepsilon$-approximation to $f$ which agrees with $f$ on $L$ is homotopic to $f$ relative to $L$.

The proof of Lees' Engulfing Theorem is now repeated, following the outline above. Care is taken that $K$ is triangulated finely enough and the coordinate neighborhoods $\left|\Psi_{i}\right|$ are chosen to have diameter $<\varepsilon$. Lees' proof produces $g: K \rightarrow M$ such that

(1) $g|L=f| L$,

(2) $g a_{i} A_{i} \subset\left|\Psi_{i}\right|, f a_{i} A_{i} \subset\left|\Psi_{i}\right|, 1 \leqq i \leqq r$,

(3) $g D=g K \subset|\Psi|$ for some coordinate neighborhood $|\Psi|$ on $Q$.

From (2) $d(f, g)<\varepsilon$ follows. From (1) and the choice of $\varepsilon, f \sim g$ rel $\dot{D}$. 
Proposition 2. If $n \leqq q-2, M^{n}$ is a topological manifold, $Q^{q}$ is a $2 n-q$ connected topological manifold and $f:(M, \dot{M}) \rightarrow(Q, \dot{Q})$ is a proper map such that, for some coordinate neighborhood $|\Psi|$ in $\stackrel{M}{M}$ and some $0<\rho^{\prime}<1, f|M-| \Psi \mid \rho^{\prime}$ is a locally flat imbedding, then for any $1>\rho>\rho^{\prime}$ there is a proper map $g: M \rightarrow Q$ such that

(1) $g=$ f off $|\Psi| \rho$,

(2) $g|\Psi| \rho \subset|\varphi|$ for some coordinate neighborhood $|\varphi|$ on $\dot{Q}$,

(3) $f \sim g$ rel $\dot{M}$.

Proof. Triangulate $C(1)$ with the subcomplex $L$ triangulating $\dot{C}(\rho)$. Then $\Psi L \subset M-|\bar{\Psi}| \rho^{\prime}$, an open set on which $f$ is a locally flat imbedding. By Lemma 4, $f \Psi \mid L$ is locally flat on every simplex. By Proposition $1, f \Psi \mid C(\rho) \sim \alpha \operatorname{rel} \dot{C}(\rho)$ via a homotopy $H: C(\rho) \times I \rightarrow Q$ where $\alpha C(\rho) \subset|\varphi|$ for some coordinate neighborhood $|\varphi|$ on $Q$, which is also $2 n-q$ connected. Define $G: M \times I \rightarrow Q$ by

$$
\begin{aligned}
G(x, t) & =H\left(\Psi^{-1} x, t\right), & & x \in|\Psi| \rho, \\
& =f(x), & & x \notin|\Psi| \rho .
\end{aligned}
$$

$G$ is well defined since if $x \in\left|\Psi^{\circ}\right| \rho, H\left(\Psi^{-1} x, t\right)=f \Psi\left(\Psi^{-1} x\right)=f x$. So $G$ is a homotopy from $f$ to a map $G_{1}=g$ which clearly satisfies (1) and (3). Finally $g|\Psi| \rho=\alpha \Psi^{-1}\left|\Psi^{\circ}\right| \rho$ $=\alpha C(\rho) \subset|\varphi|$.

The next tools to be discussed here are the general position theorems.

First General Position Theorem [6]. Let $X_{0} \subset X^{n}$ and $Y^{k}$ be polyhedra and $Q^{q}$ a P.L. manifold with $Y$ a subcomplex of $Q$. Let $f: X \rightarrow Q^{q}$ be a P.L. imbedding such that $f\left(X-X_{0}\right) \subset Q$. Then there is an arbitrarily small ambient P.L. isotopy sending $f$ into $g$ and keeping $\dot{Q} \cup f X_{0}$ fixed, such that $g \mid X-X_{0}$ is in general position with respect to $Y$. That is,

$$
\operatorname{dim} g\left(X-X_{0}\right) \cap Y \leqq n+k-q .
$$

Second General Position Theorem [6]. Let $X_{0} \subset X^{n}$ be polyhedra and $Q^{a} a$ P.L. manifold. Let $f: X \rightarrow Q$ be a map such that $f \mid X_{0}$ is a P.L. imbedding. Suppose $2 q>3(n+1)$. Then $f \sim g$ via an arbitrarily small homotopy keeping $X_{0}$ fixed such that $g$ is P.L. and in general position. That is, $\operatorname{dim} S_{2}(g) \leqq 2 n-q$ and $S_{3}(g)=\varnothing$ where $S_{r}(g)$ denotes the closure of $\left\{x \in X \mid g^{-1} g x\right.$ contains at least $r$ points $\}$.

The final tools of this section are concerned with approximating maps by P.L. maps.

TheORem of Miller [11]. Suppose $q \geqq 5, q \geqq n+3, M^{n}$ is a compact P.L. manifold, $\hat{M}^{n}$ is a compact P.L. submanifold of $M$, and $Q^{q}$ is a P.L. manifold. Let $g: M \rightarrow \dot{Q}$ be a locally flat imbedding such that $g \mid \hat{M}$ is P.L. Let $N$ be a neighborhood of $g(M-\hat{M})$ in $Q$. Then for $\varepsilon>0$, there is an ambient $\varepsilon$-isotopy $H_{t}$ of $Q$ that is fixed outside $N$ such that $H_{1} g$ is a P.L. imbedding.

Proposition 3. Suppose $q \geqq 5, q \geqq n+3, M^{n}$ and $Q^{q}$ are P.L. manifolds, and $C$ is a compact subset of $M$. Suppose $g: M \rightarrow \&$ is a map such that $S_{3}(g)=\varnothing, S_{2}(g)$ is compact, $g$ is P.L. near $S_{2}(g)$, and $g \mid M-S_{2}(g)$ is a locally flat imbedding. Then 
for any $\varepsilon>0$ there is an $\varepsilon$-homeomorphism $h: Q \rightarrow Q$, fixed on $S_{2}(g)$ and outside a compact set, such that hg is P.L. near C.

Proof. Let $N_{1}$ be a regular neighborhood of $S_{2}(g)$ near which $g$ is P.L. Let $N_{2}$ be a regular neighborhood of $N_{1}$ on which $g$ is P.L. Let $N_{3}$ be a regular neighborhood of $N_{2} \cup C$. Then apply Miller's Theorem to $M_{1}=N_{3}-\stackrel{\circ}{1}_{1}$ and $\hat{M}_{1}=N_{2}-\stackrel{\circ}{N}_{1}$. Let $h=H_{1}$.

3. The inductive argument. The first major step in the proof of this paper's main result, Theorem 1, proceeds by induction on the number of coordinate neighborhoods in a special cover of $M$. This section's first proposition isolates the inductive step.

Notation. Let $\alpha>0$ and let $\left\{\left|\Psi_{i}\right|\right\}_{i}$ be a collection of coordinate neighborhoods in $\stackrel{M}{ }$ indexed by the integers. Denote

$$
\Psi^{q}(\alpha)=\bigcup_{i \leqq q}\left|\Psi_{i}\right|_{\alpha}, \quad \Psi^{q}(\alpha)=\bigcup_{i \leqq q}\left|\Psi_{i}\right|_{\alpha} .
$$

Proposition 4. Suppose $M^{n}$ and $Q^{q}$ are topological manifolds, $M^{n}$ is $2 n-q$ connected and $q \geqq n+3$. Let $\varepsilon>0$ and $0<\alpha<1$ be given.

Suppose that for some integer $k \geqq 1, f_{k-1}: M \rightarrow Q$ is a map such that

(1) $f_{k-1}^{-1} C$ is compact for every compact subset $C$ of $Q$.

(2) There is a locally finite collection $\left\{\left|\Psi_{i}\right|\right\}_{i \leqq k}$ of coordinate neighborhoods in $\dot{M}$ and a coordinate neighborhood $|\varphi|$ in $Q$ such that $f_{k-1}\left|\bar{\Psi}_{k}\right| \subset|\stackrel{\varphi}{\varphi}|_{1}$.

(3) There are numbers $\delta$ and $\sigma$ with $\alpha<\delta<1$ and $\frac{1}{2} \leqq \sigma<1$ and a homeomorphism fixed outside a compact set $j_{k-1}: M \rightarrow M$ such that $\left.f_{k-1}\left|\Psi^{k-1}(\delta)-j_{k-1}\right| \Psi_{1}\right|_{\sigma}$ is a locally flat imbedding.

Then there is a map $f_{k}: M \rightarrow Q$, a homeomorphism $j_{k}: M \rightarrow M$ fixed outside a compact set and numbers $\beta$ and $\rho$ such that

(1) $\alpha<\beta<\delta, \sigma<\rho<1$,

(2) $j_{k}=j_{k-1}$ on $\left|\Psi_{1}\right|_{\sigma}$

(3) $f_{k}=f_{k-1}$ on $\Psi^{k-1}(\beta)-j_{k}\left|\Psi_{1}\right|_{\rho}$,

(4) $f_{k}=f_{k-1}$ off $f_{k-1}^{-1}|\varphi|_{2}$,

(5) $f_{k}^{-1} C$ is compact for every compact subset $C$ of $Q$,

(6) $\left.f_{k}\left|\Psi^{* k}(\beta)-j_{k}\right| \Psi_{1}\right|_{\rho}$ is a locally flat imbedding,

(7) $d\left(f_{k-1}, f_{k}\right)<\varepsilon$, where $d$ is the metric on $Q$ induced from a fixed imbedding of $Q$ as a closed subset of $E^{s}$ for some $s$.

The proof of Proposition 4 is very similar to the proof of Proposition 1 in [10]. The idea of the proof is the same. Technical difficulties are encountered since euclidean space $E^{q}$ is here replaced by an arbitrary manifold $Q^{q}$. The dimension requirement $q \geqq n+3$ is improved here because Miller's Theorem is now available. The details of the proof can be found in [16].

The next proposition actually accomplishes the promised inductive argument, wherein everything except one coordinate neighborhood is imbedded. The extension of the imbedding to the last neighborhood is then deferred until $\S 5$. 
Proposition 5. If $q \geqq n+3, M^{n}$ and $Q^{q}$ are topological manifolds such that $M$ is compact and $2 n-q$ connected, and $f:(M, \dot{M}) \rightarrow(Q, \dot{Q})$ is a map such that $f \mid \dot{M}$ is a locally flat imbedding, then $f \sim g$ rel $\dot{M}$ where $g: M \rightarrow Q$ is a strictly proper map and $\left.g|M-| \Psi\right|_{\rho}$ is a locally flat imbedding, where $|\Psi|$ is a coordinate neighborhood in $\dot{M}$ and $0<\rho<1$.

Proof. By Lemmas 5 and 6, after a preliminary homotopy of $f, f$ may be assumed to be strictly proper. Also it may be assumed that there is an open neighborhood $U$ of $\dot{M}$ or of some $x_{0} \in M$ if $\dot{M}=\varnothing$ such that $f \mid U$ is locally flat. Let $0<\alpha<1$ and $\left\{\left|\Psi_{i}\right| \mid i \leqq r\right\}$ be a locally finite family of coordinate neighborhoods in $M$ such that

(1) $\left\{\left|\Psi_{i}\right|_{\alpha} \mid i \leqq r\right\}$ covers $\stackrel{M}{ }$,

(2) $\Psi^{1}(1) \subset U$,

(3) $\dot{\Psi}^{1}(\alpha) \supset \bar{V}-\dot{M}$ where $\dot{M} \subset V \subset \bar{V} \subset U$ or $x_{0} \in V \subset \bar{V} \subset U$,

(4) $f\left|\bar{\Psi}_{i}\right| \subset\left|\stackrel{\circ}{i}_{i}\right|_{1}$ for some coordinate neighborhood $\left|\varphi_{i}\right|$ on $\dot{Q}$.

By induction define maps $f_{i}: M \rightarrow Q$ for $0 \leqq i \leqq r$ such that

(A) $f_{\imath} \sim f$ rel $\dot{M}$.

(B) $f_{i}\left|\bar{\Psi}_{j}\right| \subset\left|\stackrel{\circ}{\varphi}_{j}\right|_{1}$ for $j \leqq r$.

(C) There are numbers $\delta_{i}$ and $\sigma_{i}$ with $\alpha<\delta_{i}<1, \frac{1}{2} \leqq \sigma_{i}<1$ and a homeomorphism $j_{i}: M \rightarrow M$, fixed outside a compact set, such that $\left.f_{i}\left|\Psi^{i}\left(\delta_{i}\right)-j_{i}\right| \Psi_{1}\right|_{\sigma_{i}}$ is a locally flat imbedding.

Set $f_{0}=f, \alpha<\delta_{0}<1, \sigma_{0}=\frac{1}{2}, j_{0}=1_{M}$. Now suppose $f_{k-1}$ is defined for some $k \geqq 1$. Pick $\varepsilon>0$ such that

(a) $\varepsilon<\operatorname{dist}\left(f_{k-1}\left|\bar{\Psi}_{i}\right|, Q-\left|\dot{\varphi}_{i}\right|_{1}\right)$ for those finitely many indices $i$ for which $\left|\Psi_{i}\right|$ meets $f_{k-1}^{-1}\left|\varphi_{k}\right|_{2}$.

(b) Once $f_{k}$ is defined such that $f_{k}$ agrees with $f_{k-1}$ on $\dot{M}$ and $d\left(f_{k-1}, f_{k}\right)<\varepsilon$, then $f_{k} \sim f_{k-1}$ rel $\dot{M}$.

By Proposition 4, there are $f_{k}, j_{k}, \delta_{k}, \sigma_{k}$ such that $\alpha<\delta_{k}<\delta_{k-1}, \sigma_{k-1}<\sigma_{k}<1$, $d\left(f_{k-1}, f_{k}\right)<\varepsilon,\left.f_{k}\left|\Psi^{k k}\left(\delta_{k}\right)-j_{k}\right| \Psi_{1}\right|_{\sigma_{k}}$ is a locally flat imbedding. Hence (A) and (C) hold for $k$. Also $f_{k}=f_{k-1}$ on $\Psi^{k-1}\left(\delta_{k}\right)-j_{k}\left|\Psi_{1}\right|_{\sigma_{k}}$ and $f_{k}=f_{k-1}$ off $f_{k-1}^{-1}\left|\varphi_{k}\right|_{2}$. So by choice (a), $f_{k}\left|\bar{\Psi}_{i}\right| \subset\left|\stackrel{\circ}{\varphi}_{i}\right|_{1}$ for $i \leqq r$.

Finally, $g=f_{r}$ is defined along with $\alpha<\delta_{r}<1, \frac{1}{2} \leqq \sigma_{r}<1$ and $j_{r}$ such that $g \sim f$ rel $\dot{M}$. Since $\Psi^{r}\left(\delta_{r}\right) \supset \stackrel{\circ}{M},\left.g\left|\stackrel{\circ}{M}-j_{r}\right| \Psi_{1}\right|_{\sigma_{r}}$ is a locally flat imbedding. But $g=f$ on $\dot{\Psi}^{r-1}\left(\delta_{r}\right)-j_{r}\left|\Psi_{1}\right|_{\sigma_{r}}$, which is a deleted neighborhood of $\dot{M}$. Hence, $g=f$ near $\dot{M}$ and $g$ is locally flat on all of $M-j_{r}\left|\Psi_{1}\right|_{\sigma_{r}}$.

4. Connectivity considerations. This short section considers various connectivity statements which are of use in this paper. The most important of these statements is Lemma 9, which is crucial to the completion of the proof of this paper's main result.

Lemma 7. Let $Q^{q}$ be a topological manifold without boundary, $q \geqq 5$. Let $A$ be $a$ closed subset of $Q$. Let $C$ be a closed $n$-cube where $q \geqq n+3$. Suppose $f: C \rightarrow Q$ is an imbedding which is locally flat on every simplex in some triangulation of $C$. Then any 
map $\alpha: S^{1} \rightarrow Q-(A \cup f C)$ which is null-homotopic in $Q-A$ is null-homotopic in $Q-(A \cup f C)$.

Proof. Since $q>2 \operatorname{dim} S^{1}+2$, after a preliminary homotopy inside $Q-(A \cup f C)$, $\alpha$ can be assumed to be a locally flat imbedding [2]. It then must be shown that $\alpha$ extendstoa map $D^{2} \rightarrow Q-(A \cup f C)$. At any rate, $\alpha$ extends to a map $\beta: D^{2} \rightarrow Q-A$. By Proposition 2, $\beta \sim \beta^{\prime}$ rel $S^{1}$ so that $\beta^{\prime} D^{2} \subset|\stackrel{\varphi}{\varphi}|_{1}$ for some coordinate neighborhood $|\varphi|$ in $Q-A$.

By Miller's Theorem, there is an $\varepsilon$-homeomorphism $\bar{h}: E^{q} \rightarrow E^{q}$ fixed outside the $\varepsilon$-neighborhood of $\varphi^{-1} \alpha S^{1}$ such that $\bar{h} \varphi^{-1} \alpha$ is P.L. Now $\bar{h}$ defines a homeomorphism $h: Q \rightarrow Q$. Then $\varphi^{-1} h \alpha$ is P.L., and if $\varepsilon$ is small enough $\operatorname{Im} h \beta^{\prime} \subset|\stackrel{\circ}{\varphi}|_{1}$ and Im $h \alpha \subset Q-(A \cup f C)$.

Triangulate $C$ finely enough that $h f$ is locally flat on every simplex and for no simplex $\sigma$ does $h f \sigma$ meet both $|\dot{\varphi}|_{2}$ and $|\dot{\varphi}|_{1}$. Then $C=K \cup J$ where

$$
K=\left\{\left.\sigma|h f \sigma \subset| \stackrel{\circ}{\varphi}\right|_{2}\right\}, \quad J=\left\{\left.\sigma|h f \sigma \cap| \varphi\right|_{1}=\varnothing\right\} .
$$

By Miller's Theorem again there is an $\varepsilon$-homeomorphism $\bar{k}: E^{q} \rightarrow E^{q}$ fixed outside the $\varepsilon$-neighborhood of $\varphi^{-1} h f K$ such that $\bar{k} \varphi^{-1} h f$ is a P.L. imbedding near $K . \bar{k}$ defines $k: Q \rightarrow Q$ so that $\varphi^{-1} k h f \mid K$ is P.L. If $\varepsilon$ is small enough $\operatorname{Im} k h \beta^{\prime} \subset|\dot{\varphi}|_{1}$, $k h f J \cap|\varphi|_{1}=\varnothing$, and $k$ is fixed on $h \alpha S^{1}$, which misses $h f C$. Thus, $\varphi^{-1} k h \beta^{\prime} \mid S^{1}$ $=\varphi^{-1} h \alpha$ is a P.L. imbedding and $\varphi^{-1} k h \beta^{\prime} \sim \theta$ rel $S^{1}$ by an $\varepsilon$-homotopy where $\theta: D^{2} \rightarrow E^{q}$ is P.L. in general position with respect to $\varphi^{-1} k h f K$. Then

$$
\begin{gathered}
\operatorname{dim}(\operatorname{Im} \theta) \cap \varphi^{-1} k h f K \leqq 2+n-q<0, \\
\operatorname{Im} \theta \cap \varphi^{-1} k h f K=\varnothing, \text { and } \operatorname{Im} \varphi \theta \cap k h f K=\varnothing .
\end{gathered}
$$

If $\varepsilon$ is small enough, $\operatorname{Im} \varphi \theta \subset|\stackrel{\varphi}{ }|_{1}$. Since $k h f J \cap|\varphi|_{1}=\varnothing, \operatorname{Im} \varphi \theta \cap k h f C=\varnothing$ and so $\operatorname{Im} h^{-1} k^{-1} \varphi \theta \cap f C=\varnothing$. Since $h$ and $k$ are fixed outside $|\varphi|$ and $|\varphi| \cap A=\varnothing$, it follows that $\operatorname{Im} \theta h^{-1} k^{-1} \varphi \cap(A \cup f C)=\varnothing$. Thus, $h^{-1} k^{-1} \varphi \theta: D^{2} \rightarrow Q-(A \cup f C)$ and $h^{-1} k^{-1} \varphi \theta\left|S^{1}=h^{-1} k^{-1} \varphi \varphi^{-1} k h \beta^{\prime}\right| S^{1}=\alpha$.

The proof of the important Lemma 9 uses homology and cohomology functors which are defined in sheaf theory. For the relevant definitions and notation see [15], especially Chapter XI.

LEMmA 8. Let $f: X \rightarrow Y$ be a homeomorphism. Let $G$ be a sheaf over $Y$ and $\Phi$ a family of supports in $Y$. Let $f^{-1} \Phi=\left\{f^{-1} A \mid A \in \Phi\right\}$ and let $f^{*} G$ be the sheaf over $X$ induced by $f$. Then $f$ induces an isomorphism of augmented $\delta$-functors

$$
H_{\Phi}^{*}(Y, G) \rightarrow H_{f-1}^{*}\left(X, f^{*} G\right) .
$$

Proof. $f$ and $f^{-1}$ induce unique maps of augmented $\delta$-functors

$$
H_{\Phi}^{*}(Y, G) \leftrightarrows H_{f-1 \Phi}^{*}\left(X, f^{*} G\right)
$$

Since $G=\left(f^{-1}\right)^{*} f^{*} G$ and both compositions are the unique maps induced by the identity, the isomorphism follows. 
LeMma 9. Let $q \geqq n+3, M^{n} a 2 n-q$ connected topological manifold and $Q^{q} a$ simply connected topological manifold. Suppose $f: M \rightarrow Q$ is a strictly proper map such that for some coordinate neighborhood $|\Psi|$ on $\left.\stackrel{M}{M} f|M-| \Psi\right|_{1}$ is a locally flat imbedding. Then $\pi_{i}\left(Q, Q-f\left(M-|\Psi|_{2}\right)\right)=0$ for $i \leqq n$.

Proof. Since $f$ is strictly proper, the pairs $\left(Q, Q-f\left(M-|\Psi|_{2}\right)\right)$ and $\left(\stackrel{Q}{Q}, \dot{Q}-f\left(\stackrel{M}{M}-|\dot{\Psi}|_{2}\right)\right)$ are homotopy equivalent. It may then be assumed that $\dot{M}=\dot{Q}=\varnothing$.

Let $S_{Q}$ denote the sheaf of singular chains on $Q$ and $T_{Q}=H_{q}\left(S_{Q}\right)$. Similarly, $T_{M}=H_{n}\left(S_{M}\right)$. Since $Q$ is simply connected, it is orientable and $T_{Q}$ is the constant sheaf with stalk $Z$. Let $Z_{X}$ denote the constant sheaf over any space $X$ with stalk $Z$. Let $A=M-|\Psi|_{2}$. Then $(f \mid A)^{*}\left(T_{Q} \mid f A\right)=Z_{A}$. Let $\Phi_{M}$ and $\Phi_{Q}$ denote the paracompactifying families of compact subsets of $M$ and $Q$ respectively. Then

$\Phi_{f A}=\left\{B \in \Phi_{Q} \mid B \subset f A\right\}=$ set of compact sets in $f A$,

$\Phi_{A}=\left\{B \in \Phi_{M} \mid B \subset A\right\}=$ set of compact sets in $A$, and $f^{-1} \Phi_{f A}=\Phi_{A}$.

Then by the duality theorems in [15], Lemma 8 , and obvious isomorphisms

$$
\begin{aligned}
H_{i}(Q, Q-f A) & \cong H_{i}^{\Phi_{Q}}\left(Q, Q-f A ; Z_{Q}\right) \cong H_{\Phi_{f A}}^{q-i}\left(f A ;\left(T_{Q} \otimes Z_{Q}\right) \mid f A\right) \\
& \cong H_{f-1_{\Phi_{A}}^{q-i}}^{q}\left(A ;(f \mid A)^{*}\left(T_{Q} \mid f A\right)\right) \cong H_{\Phi_{A}}^{q-i}\left(A ; Z_{A}\right) .
\end{aligned}
$$

Suppose $2 n-q \leqq 0$. Then for $i \leqq n, q-i \geqq q-n \geqq n$, and

$$
H_{\Phi_{A}}^{q-i}\left(A ; Z_{A}\right) \cong H^{q-i}(A ; Z)=0 .
$$

Suppose $2 n-q \geqq 1$. Then $M$ and $A$ are orientable and $Z_{A} \cong T_{M}\left|A \cong\left(T_{M} \otimes Z_{M}\right)\right| A$. Then by more duality arguments:

$$
\begin{aligned}
H_{\Phi_{A}}^{q-i}\left(A ; Z_{A}\right) & \cong H_{\Phi_{A}^{q-i}}^{q}\left(A ;\left(T_{M} \otimes Z_{M}\right) \mid A\right) \cong H_{n}^{\Phi_{M}(q-i)}\left(M, M-A ; Z_{M}\right) \\
& \cong H_{n-(q-i)}\left(M,|\Psi|_{2}\right) \cong \tilde{H}_{n-(q-i)}(M)=0
\end{aligned}
$$

for $n-(q-i) \leqq 2 n-q$, i.e., for $i \leqq n$.

So in either case $H_{i}(Q, Q-f A)=0$ for $i \leqq n$. The lemma then follows from the relative Hurewicz Theorem provided it is shown that $Q-f A$ is simply connected. It is certainly arcwise connected by dimension theory. Now there is a locally finite cover of $A$ by coordinate neighborhoods $\left|\Psi_{1}\right|,\left|\Psi_{2}\right|, \ldots$ in $M-|\Psi|_{1}$ such that $A \subset\left|\Psi_{1}\right|_{1} \cup\left|\Psi_{2}\right|_{1} \cup \ldots$. Then $\left\{f\left|\Psi_{i}\right|_{1} \mid i \geqq 1\right\}$ is locally finite in $Q$. Let $M_{k}$ $=\bigcup_{i \geqq k} f\left|\Psi_{i}\right|_{1}$. Then $M_{k}$ is closed in $Q$.

Let $\alpha: S^{1} \rightarrow Q-f A$ be given. Since $Q$ is simply connected, $\alpha$ is null-homotopic in $Q$ via a homotopy $H$. Now $\operatorname{Im} H$ intersects $f\left|\Psi_{i}\right|_{1}$ for only finitely many values of $i$. Hence, Im $H \subset Q-M_{k}$ for some $k$ and $\alpha$ is null-homotopic in $Q-M_{k}$. Lemma 7 can now be applied finitely many times to show that $\alpha$ is null-homotopic in $Q-M_{1} \subset Q-f A$.

5. The imbedding theorem. The proof of the main result of this paper can now be accomplished. The opening part of this section consists of a moderately involved argument which reduces the proof to a special case. The special case is then 
proved by means of an intricate argument combining most of the topological and P.L. techniques considered in the previous sections. Finally, some corollaries are included.

THEOREM 1. If $q \geqq n+3, M^{n}$ is a compact, $2 n-q$ connected topological manifold, $Q^{q}$ is a $2 n-q+1$ connected topological manifold and $f:(M, \dot{M}) \rightarrow(Q, \dot{Q})$ is a map such that $f \mid \dot{M}$ is a locally flat imbedding, then $f \sim g$ rel $\dot{M}$ where $g: M \rightarrow Q$ is a proper locally flat imbedding.

Proof. By Proposition 5, after a preliminary homotopy, it can be assumed that $f$ is strictly proper and $\left.f|M-| \Psi\right|_{\rho^{\prime}}$ is a locally flat imbedding for some coordinate neighborhood $|\Psi|$ in $\stackrel{M}{M}$ and some $1>\rho^{\prime}>0$. Pick $\rho^{\prime}<\rho<1$. By Proposition 2, $f \sim f_{1}$ rel $\dot{M}$ where $f_{1}=f$ off $|\dot{\Psi}|_{\rho}$ and $f_{1}|\Psi|_{\rho} \subset|\varphi|$ for some coordinate neighborhood $|\varphi|$ on $Q$. By choosing $\rho$ slightly larger it may be assumed that $f_{1}$ is a locally flat imbedding near $M-|\dot{\Psi}|_{\rho}$.

Pick $\rho<\sigma<1$ such that $f_{1}|\Psi|_{\sigma} \subset|\varphi|$. Let $C(\sigma)$ be triangulated so that $\dot{C}(\rho), C(\rho)$, and $L=C(\sigma)-\stackrel{\circ}{C}(\rho)$ are triangulated as subcomplexes. Now

$$
\varphi^{-1} f_{1} \Psi \mid L: L \rightarrow E^{q}
$$

is an imbedding which is locally flat on every simplex. By Miller's Theorem, there is an $\varepsilon$-homeomorphism $\bar{h}: E^{q} \rightarrow E^{q}$ fixed outside the $\varepsilon$-neighborhood of $\varphi^{-1} f_{1} \Psi L$ such that $\bar{h} \varphi^{-1} f \Psi \mid L$ is a P.L. imbedding. Now if $\varepsilon$ is small enough, $\bar{h}$ defines an $\varepsilon$-homeomorphism $h: Q \rightarrow Q$. Then $f_{2}=h f_{1}$ has the same properties as $f_{1}$ and $f_{1} \sim f_{2}$ rel $\dot{M}$ if $\varepsilon$ is small enough.

Pick $\sigma>\tau_{1}>\tau_{2}>\tau>\rho$ and suppose $C(\sigma)$ is further subdivided so that $\dot{C}\left(\tau_{1}\right)$, $\dot{C}\left(\tau_{2}\right)$, and $\dot{C}(\tau)$ are triangulated as subcomplexes. Now $k=\varphi^{-1} f_{2} \Psi\left|L=\varphi^{-1} h f_{1} \Psi\right| L$ $=\bar{h} \varphi^{-1} f_{1} \Psi \mid L$ is a P.L. imbedding. Triangulate $L$ and $E^{q}$ such that $k$ is simplicial and if $A=N\left(\dot{C}(\tau), L^{\prime \prime}\right)$ is the simplicial neighborhood in the second derived subdivision $L^{\prime \prime}$ of $L$, then $A \cap\left(C(\sigma)-\dot{C}\left(\tau_{1}\right)\right)=\varnothing$. Let $D=N\left(k \dot{C}(\tau),\left(E^{q}\right)^{\prime \prime}\right)$ be the corresponding simplicial neighborhood in the second derived subdivision of $E^{q}$. Then $k$ imbeds $A$ properly into $D$ and $k^{-1} D=A$. Now $\left(C(\sigma)-\dot{C}\left(\tau_{1}\right)\right) \cup A$ and $\left[C(\sigma)-\dot{C}\left(\tau_{1}\right)\right] \cup\left[C\left(\tau_{2}\right)-\dot{C}(\rho)\right]$ are regular neighborhoods of $\dot{C}(\sigma) \cup \dot{C}(\tau)$ in $C(\sigma)$. By the uniqueness of regular neighborhoods there is a P.L. homeomorphism $l$ of $C(\sigma)$ fixed on $\dot{C}(\sigma) \cup \dot{C}(\tau)$ such that

$$
l\left(\left[C(\sigma)-\stackrel{\circ}{C}\left(\tau_{1}\right)\right] \cup A\right)=\left[C(\sigma)-\dot{C}\left(\tau_{1}\right)\right] \cup\left[C\left(\tau_{2}\right)-\stackrel{\circ}{C}(\rho)\right] .
$$

Then

$$
k_{1}=k\left(l^{-1}\right): L, C(\tau)-C(\rho), \dot{C}\left(\tau_{2}\right) \cup \dot{C}(\rho) \rightarrow E^{q}, \dot{D}, \dot{D}
$$

is a P.L. imbedding and $k_{1}=k$ on $\dot{C}(\sigma)$. Extend $k_{1}$ to $C(\sigma)$ by means of any extension of $k_{1} \mid \dot{C}(\rho)$ to $C(\rho)$. Define $f_{2}: M \rightarrow Q$ by

$$
\begin{aligned}
f_{2} & =f_{1} & & \text { on } M-|\Psi|_{\sigma}, \\
& =\varphi k_{1} \Psi^{-1} & & \text { on }|\Psi|_{\sigma} .
\end{aligned}
$$


Note that if $x \in|\dot{\Psi}|_{\sigma}, \varphi k_{1} \Psi^{-1} x=\varphi k \Psi^{-1} x=f_{1} x$. Hence $f_{2}$ is well defined. Since their images disagree only in the coordinate neighborhood $|\varphi|, f_{2} \sim f_{1}$ rel $\dot{M}$. Note that $\varphi^{-1} f_{2} \Psi \mid L$ is a P.L. imbedding.

The remainder of the proof is divided into two cases.

Case $1 . Q$ is not simply connected.

Then $2 n-q+1<1$ and $2 n<q$. Now $M_{1}=f_{2}^{-1}|\varphi|$ is an open $n$-submanifold of $M$, and $M^{*}=\varphi^{-1} f_{2}\left(M_{1}-|\Psi|_{\tau}\right)$ is closed in $E^{q}$ since $\varphi M^{*}=f_{2}\left(M-|\Psi|_{\tau}\right) \cap|\varphi|$. Then $R_{1}=E^{q}-\left(M^{*} \cup \dot{D}\right)$ is a P.L. $q$-manifold with boundary $\dot{D}-M^{*}$. By collapsing $D$ onto $k_{1} \dot{C}(\tau)=\varphi^{-1} f_{2}|\dot{\Psi}|_{\tau}, R_{1}$ and $E^{q}-M^{*}$ are seen to be homotopy equivalent. Hence, $\pi_{i}\left(E^{q}-M^{*}, R_{1}\right)=0$ for all $i$. By Lemma $9, \pi_{i}\left(E^{q}, E^{q}-M^{*}\right)=0$ for $i \leqq n$. So from the exact homotopy sequence of the triple $\left(E^{q}, E^{q}-M^{*}, R_{1}\right)$ it follows that $\pi_{i}\left(E^{q}, R_{1}\right)=0$ for $i \leqq n$.

Now $k_{1} \mid C(\rho):(C(\rho), \dot{C}(\rho)) \rightarrow\left(E^{q}, R_{1}\right)$. So $k_{1} \mid C(\rho) \sim k_{2}$ rel $\dot{C}(\rho)$ where

$$
k_{2}: C(\rho) \rightarrow R_{1} \text {. }
$$

Now $k_{2}\left|\dot{C}(\rho)=k_{1}\right| \dot{C}(\rho)$ is a P.L. imbedding into $\dot{R}_{1}$. Since $2 n<q$, the pairs $(C(\rho)$, $\dot{C}(\rho))$ and $\left(R_{1}, \dot{R}_{1}\right)$ are certainly $2 n-q$ and $2 n-q+1$ connected, respectively. By Irwin's Imbedding Theorem, $k_{2} \mid C(\rho) \sim k_{3}$ rel $C(\rho)$ where $k_{3}: C(\rho) \rightarrow R_{1}$ is a proper P.L. imbedding. Extend $k_{3}$ to $C(\sigma)$ by setting $k_{3}=k_{2}$ off $C(\rho)$. Then $k_{3}: C(\sigma) \rightarrow E^{q}$ is a P.L. imbedding and is locally flat by Lemma 3. Define $g: M \rightarrow Q$ by

$$
\begin{aligned}
g & =\varphi k_{3} \Psi^{-1} & & \text { on }|\Psi|_{\sigma}, \\
& =f_{2} & & \text { off }|\Psi|_{\sigma} .
\end{aligned}
$$

$g$ is well defined for if $x \in|\dot{\Psi}|_{\sigma}, \varphi k_{3} \Psi^{-1} x=\varphi k_{1} \Psi^{-1} x=f_{2} x . g$ is locally flat since it is locally flat on $|\Psi|_{\sigma}$ and off $|\Psi|_{\tau}$. Thus $g$ is a locally flat imbedding, and $g \sim f_{2}$ rel $\dot{M}$ since their images disagree only inside $|\varphi|$.

Case 2. $Q$ is simply connected.

Let $R=Q^{\circ}-\left(M^{\prime} \cup \varphi D^{\circ}\right)$ where $M^{\prime}=f_{2}\left(M-|\dot{\Psi}|_{\tau}\right)$.

Claim. $\pi_{\imath}(\ell, R)=0$ for $i \leqq n$.

As in Case 1, the collapse of $D$ onto $k_{1} \dot{C}(\tau)$ implies that $\pi_{i}\left(Q^{\prime}-M^{\prime}, R\right)=0$ for all $i$. By Lemma $9, \pi_{i}\left(Q, Q-M^{\prime}\right)=\pi_{i}\left(Q, Q-M^{\prime}\right)=0$ for $i \leqq n$. The claim follows from the exact homotopy sequence of the triple $\left(Q, Q-M^{\prime}, R\right)$.

Since $n+3 \leqq q$, it follows that $2 n-q+2 \leqq n-1$. Since $Q$ is $2 n-q+1$ connected, it follows from the homotopy sequence of $(Q, R)$ that $R$ is $2 n-q+1$ connected.

Since $\left.f_{2}|| \Psi\right|_{\rho}:\left(|\Psi|_{\rho},|\dot{\Psi}|_{\rho}\right) \rightarrow(\ell, R),\left.f_{2}|| \Psi\right|_{\rho} \sim f_{3}:|\Psi|_{\rho} \rightarrow R$ rel $|\dot{\Psi}|_{\rho}$. $f_{3}$ extends to $M$ by setting $f_{3}=f_{2}$ off $|\Psi|_{\rho}$. Then $f_{3} \sim f_{2}$ rel $\dot{M}$ and

$$
\left.f_{3}|| \Psi\right|_{\rho}:\left(|\Psi|_{\rho},|\dot{\Psi}|_{\rho}\right) \rightarrow(R, \dot{R}) \text {. }
$$

Now $\dot{R}=\varphi\left(\dot{D}-k_{1} \dot{C}\left(\tau_{1}\right)\right)$ is a P.L. manifold via the P.L. structure of $\dot{D}-k_{1} \dot{C}\left(\tau_{1}\right)$. Now $|\Psi|_{\sigma}$ has a P.L. structure via $\Psi$, and $\dot{R}$ has neighborhoods in $Q$ and $R$ which have P.L. structures via $\varphi$. With respect to these P.L. structures, $\left.f|| \Psi\right|_{\sigma}-|\Psi|_{\rho}$ is a P.L. imbedding. 
Suppose for the moment that $\left.f_{3}|| \Psi\right|_{\rho} \sim g$ rel $|\dot{\Psi}|_{\rho}$ where $g:|\Psi|_{\rho} \rightarrow R$ is a proper locally flat imbedding which is P.L. near $|\dot{\Psi}|_{\rho}$ in $|\Psi|_{\rho}$. Then extend $g$ to $M$ by setting $g=f_{3}$ off $|\dot{\Psi}|_{\rho}$. Then clearly $g$ is a proper imbedding and $g \sim f_{3} \sim f$ rel $\dot{M}$. $g$ is clearly locally flat on $M-|\Psi|_{\rho}$ and on $|\Psi|_{\rho}$. Since $g$ is a P.L. imbedding near $|\dot{\Psi}|_{\rho}$, it is locally flat there by Lemma 3. So $g$ is locally flat everywhere.

So the proof of Theorem 1 has been reduced to the proof of the following special case.

Proposition 6. Suppose that $q \geqq n+3, D^{n}$ is a P.L. $n$-disk, $R^{q}$ is a $2 n-q+1$ connected topological manifold, and $\dot{R}$ is a P.L. manifold. Then if $f:(D, \dot{D}) \rightarrow(R, \dot{R})$ is a map such that $f \mid \dot{D}$ is a P.L. imbedding, $f \sim g$ rel $\dot{D}$ where $g: D \rightarrow R$ is a proper locally flat imbedding and $g$ is P.L. near $\dot{D}$ with respect to some P.L. structure near $\dot{R}$ which is compatible with the given P.L. structure on $\dot{R}$.

Proof. Pick $0<t<1$ and let $D_{0}=\{x \in D|| x \mid \leqq t\}$ and $W=\{x \in D|| x \mid>t\}$.

By Lemma 5, after a preliminary homotopy, $f$ may be assumed to be strictly proper with respect to collar neighborhoods $W$ and $U$ in $D$ and $R$ respectively, and $f \mid W$ is a locally flat imbedding. $\bar{W}$ can be taken to be a P.L. subspace of $D$.

The map $f$ will be changed several additional times during this proof. In each case the change will be effected by a proper homotopy, but mention of this fact will usually be omitted. For example, by Proposition 2 it may be assumed that $f$ is changed so that $f D_{0} \subset|\varphi|$ for some coordinate neighborhood $|\varphi|$ in $R$.

Let $|\varphi|$ have the P.L. structure induced from $E^{q}$ by $\varphi$. Let $N$ be a compact neighborhood of $f D_{0}$ in $|\varphi|$. Let $X$ be a finite polyhedron such that $f^{-1} N \subset X \subset f^{-1}|\varphi|$. Let $P_{1}$ be a regular neighborhood of $P_{0}=X \cap \bar{W}$ in $W \cap f^{-1}|\varphi|$. Then $f \mid P_{1}$ is locally flat. By Miller's Theorem, there is a small enough homeomorphism $h$ of $|\varphi|$ which is fixed outside a neighborhood of $f P_{1}$, and is such that $h f \mid P_{1}$ is a P.L. imbedding into $|\varphi|$. $h$ extends by the identity to a homeomorphism of $R$. Rename $h f$ to be $f$.

Let $0<\varepsilon<\operatorname{dist}\left(f D_{0},|\varphi|-N\right)$. Let $P=P_{0} \cup D_{0}$. By the Second General Position Theorem, $f \mid P \sim \eta$ rel $P_{0}$ via an $\varepsilon$-homotopy, where $\eta$ is a P.L. map in general position. That is $S_{3}(\eta)=\varnothing$ and $\operatorname{dim} S_{2}(\eta) \leqq 2 n-q$. Define $f^{\prime}: D \rightarrow R$ by

$$
\begin{aligned}
f^{\prime} & =\eta \quad \text { on } P, \\
& =f \text { on } \bar{W} .
\end{aligned}
$$

$f^{\prime}$ is well defined since $P \cap \bar{W} \subset P_{0}$ and $f=\eta$ on $P_{0}$. Then $f^{\prime}$ is P.L. on $P_{2}=P \cup P_{1}$.

Claim 1. $S_{2}\left(f^{\prime}\right)=S_{2}\left(f^{\prime} \mid P\right)$.

Let $x, y \in D, x \neq y, f^{\prime} x=f^{\prime} y$ and suppose $x \notin P$. Then $x \in W$ and since $f^{\prime} \mid W$ is an imbedding, $y \notin W$. Then $y \in D_{0}$. Now $f^{\prime} y=f^{\prime} x=f x$. So $d(f x, f y)=d\left(f^{\prime} y, f y\right)$ $=d(\eta y, f y)<\varepsilon$. By choice of $\varepsilon, f x \in N$ and $x \in X \cap \bar{W}=P_{0} \subset P$. This is a contradiction.

If $f^{\prime}$ is renamed $f$, then $S_{2}(f)$ is a P.L. subspace of $D^{\circ}$ of dimension $\leqq 2 n-q$. By Theorem 7.7 in [6], $S_{2}(f) \subset C$ where $C$ is a collapsible P.L. subspace in $D^{\circ}$ of dimension $\leqq 2 n-q+1$. 
Claim 2. It may be assumed $f C \subset|\varphi|$. (This is not a priori true.)

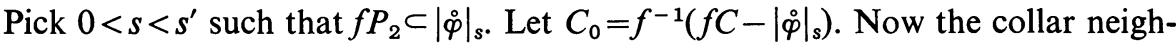
borhood $U$ inherits a P.L. structure from $\dot{R} \times[0,1]$. The map $f \mid W$ is locally flat. By Proposition 3, there is a homeomorphism $u: U \rightarrow U$ such that $u f \mid W$ is P.L. There are triangulations such that $u f \mid f^{-1}\left(f C-|\varphi|_{s}\right)$ is a simplicial map into $U$. By considering stars in $U$ of vertices in $u\left(f C-|\varphi|_{s}\right)$, it is seen that $u\left(f C-|\varphi|_{s}\right)$ and $f C-|\varphi|_{s}$ are locally tame of dimension $\leqq 2 n-q+1$.

Now $\stackrel{\circ}{R}-|\varphi|_{s}$ and $|\stackrel{\varphi}{\varphi}|_{s^{\prime}}-|\varphi|_{s}$ are $2 n-q+1$ connected and $f C-\mid \stackrel{\circ}{\varphi_{s^{\prime}}}$ is compact. By Newman's Engulfing Theorem, there is a homeomorphism $k$ of $\stackrel{R}{R}-|\varphi|_{s}$ onto itself such that $k$ is fixed outside a compact set and $k\left(\left|\varphi^{\circ}\right|_{s^{\prime}}-|\varphi|_{s}\right) \supset f C-|\varphi|_{s}$. Extend $k$ by the identity to a homeomorphism of $R$. Then $k \varphi$ defines a new coordinate neighborhood which can be renamed $|\varphi|$. Then $f C \subset|\varphi|_{s^{\prime}}$. Since $k$ is fixed on $|\varphi|_{s}$, $f \mid P_{2}$ is P.L. as a map into the new $|\varphi|$.

By Lemma $3, f$ is locally flat on $P_{2}-S_{2}(f)$. Also $f \mid W$ is locally flat. So if $M_{0}$ $=f^{-1}|\varphi|$, then $f$ is locally flat on $M_{0}-S_{2}(f)$.

Claim 3. It may be assumed that $f$ is P.L. near $C$.

$f \mid M$ is P.L. near $P$. By Proposition 3 there is a homeomorphism $h$ of $|\varphi|$ fixed outside a compact set such that $h f \mid M_{0}$ is P.L. near $C$. $h$ extends to a homeomorphism of $R$. Let $h f$ be renamed $f$.

By Theorem 7.7 in [6], $f C \subset K$ where $K$ is a collapsible P.L. subspace of $|\varphi|$ of dimension $\leqq 2 n-q+2$.

Claim 4. It may be assumed that there is a compact P.L. neighborhood $T$ of $f^{-1} K$ on which $f$ is P.L.

Let $F$ be a compact neighborhood of $K \cap f D$ in $|\varphi|$. Let $0<\alpha<\operatorname{dist}(K, f D-F)$. By Proposition 3 there is an $\alpha$-homeomorphism $h$ of $|\varphi|$ fixed outside a compact set such that $h f \mid M_{0}$ is P.L. near $f^{-1} F$. Extend $h$ to a homeomorphism of $R$. Let $T$ be a compact P.L. neighborhood of $f^{-1} F$ on which $h f$ is P.L. Let $x \in(h f)^{-1} K$. Then $h f x \in K, d(h f x, f x)<\alpha, f x \in F$, and $x \in f^{-1} F \subset \stackrel{\circ}{T}$. So $(h f)^{-1} K \subset \stackrel{\circ}{T}$. If $h f$ is renamed $f$, then Claim 4 is established.

Let $0<\beta<\operatorname{dist}(K, f D-f \stackrel{\circ}{T})$. By the First General Position Theorem, there is a P.L. $\beta$-homeomorphism $h$ of $|\varphi|$ fixed on $f C$ such that $h(K-f C)$ is in general position with respect to $f T$. Then $\operatorname{dim} h(K-f C) \cap f T \leqq 2 n-q+2+n-q \leqq 2 n-q-1$.

Claim 5. $h(K-f C) \cap f D \subset f \stackrel{\circ}{\text {. }}$

Suppose $y \in K-f C, x \in D$ and $h y=f x$. Then $d(y, f x)=d(y, h y)<\beta$. Thus $h y \in$ $f \stackrel{\circ}{T}$, which establishes Claim 5 .

Let $K_{1}=h K, C_{1}=C$. Then it follows from Claim 5 and $h \mid f C=1$ that $\left(K_{1}-f C\right) \cap f D \subset f \stackrel{\circ}{T}$ and $\operatorname{dim}\left(K_{1}-f C_{1}\right) \cap f D \leqq 2 n-q-1$. Then $f^{-1} K_{1}=C_{1} \cup X_{1}$ where $\operatorname{dim} X_{1} \leqq 2 n-q-1$. Since $f X_{1} \subset f \stackrel{\circ}{T}$ and $S_{2}(f) \subset \stackrel{\circ}{T}$, it follows that $X_{1} \subset \stackrel{\circ}{T}$. So $f$ is P.L. near $C_{1} \cup X_{1}$.

Now suppose by induction there are collapsible P.L. subspaces $C_{i} \subset \grave{D}$ and $K_{i} \subset|\varphi|$ and also a P.L. subspace $X_{i} \subset D^{\circ}$ such that $S_{2}(f) \subset C_{i}, f^{-1} K_{i}=C_{i} \cup X_{i}, f$ is P.L. near $C_{i} \cup X_{i}$ as a map into $|\varphi|$, and $\operatorname{dim} X_{i} \leqq 2 n-q-i \leqq n-3$. 
By Theorem 7.7 in [6] there is a collapsible P.L. subspace $C_{i+1} \subset D$ with $C_{i} \cup X_{i} \subset C_{i+1}$ and $\operatorname{dim}\left(C_{i+1}-C_{i}\right) \leqq \operatorname{dim} X_{i}+1$. As in Claims 2 and 3, it may be assumed that $f C_{i+1} \subset|\varphi|$ and $f$ is P.L. near $C_{i+1}$. Again by Theorem 7.7 in [6], there is a collapsible P.L. subspace $K$ of $|\varphi|$ such that $K_{i} \cup f C_{i+1} \subset K$ and $\operatorname{dim}\left(K-K_{i}\right) \leqq \operatorname{dim} X_{i}+2 \leqq 2 n-q-i+2$. As in Claim 4, it may be assumed that there is a compact P.L. neighborhood $T$ of $f^{-1} K$ on which $f$ is P.L.

Let $0<\beta<\operatorname{dist}\left(K, f D-f T^{\circ}\right)$. By the First General Position Theorem there is a P.L. $\beta$-homeomorphism $h$ of $|\varphi|$ fixed on $f C_{i+1}$ such that

$$
\operatorname{dim} h\left(K-f C_{i+1}\right) \cap f T \leqq 2 n-q-i+2+n-q \leqq 2 n-q-(i+1) .
$$

Let $K_{i+1}=h K$. Then as before $f^{-1} K_{i+1}=C_{i+1} \cup X_{i+1}$ where $\operatorname{dim} X_{i+1} \leqq 2 n-q-$ $(i+1)$ and $f$ is P.L. near $X_{i+1} \cup C_{i+1}$. So for some integer $k, X_{k}=\varnothing$ and $f^{-1} K_{k}=C_{k}$.

Let $V$ be a regular neighborhood of $C_{k}$ in $D^{\circ}$ on which $f$ is P.L. as a map into $|\varphi|$. Let $V$ and $|\varphi|$ be given second derived subdivisions of some triangulations in which $f \mid V$ is simplicial. Let $B_{1}=N\left(C_{k}, V\right)$ and $B_{2}=N\left(K_{k},|\varphi|\right)$ be the corresponding simplicial neighborhoods. Since $C_{k}$ and $K_{k}$ are collapsible, $B_{1}$ and $B_{2}$ are P.L. balls of dimension $n$ and $q$ respectively in $D^{\circ}$ and $B_{1}=f^{-1} B_{2}$ and $S_{2}(f) \subset B_{1}$.

Since $f \mid \dot{B}_{1}$ is a P.L. imbedding into $\dot{B}_{2}$, it extends to a proper P.L. imbedding $g: B_{1} \rightarrow B_{2}$. Finally, $g$ extends to $D$ by setting $g=f$ off $B_{1}$. Then $g$ is an imbedding since it has no double points. $g$ is locally flat near $B_{1}$ by Lemma 3. Hence, $g$ is a locally flat imbedding and $g \sim f$ rel $\dot{D}$.

The next result follows directly from Theorem 1 .

THEOREM 2. If $q \geqq n+3, M^{n}$ is a closed $2 n-q$ connected topological manifold, $Q^{q}$ is a $2 n-q+1$ connected topological manifold, and $f: M \rightarrow Q$ is any map, then $f \sim g$ where $g: M \rightarrow Q$ is a locally flat imbedding.

COROLlaRY. If $Q^{q}$ is a $k$-connected topological manifold, every element of $\pi_{r}(Q)$ is representable by a locally flatly imbedded sphere provided

$$
r \leqq \min (q-3,(k+q-1) / 2) .
$$

Proof. Theorem 2 and the inequalities $q \geqq r+3$ and $2 r-q+1 \leqq k$.

THEOREM 3. If $q \geqq n+3, M^{n}$ is a closed $2 n-q+1$ connected topological manifold, $Q^{a}$ is a $2 n-q+2$ connected topological manifold, and $f, g: M \rightarrow Q$ are homotopic locally flat imbeddings of $M$ in $Q$, then $f$ and $g$ are locally flatly concordant. That is, there is a locally flat imbedding $H: M \times I \rightarrow Q \times I$ such that $H(x, 0)=(f x, 0)$ and $H(x, 1)=(g x, 1)$.

Proof. Let $F: M \times I \rightarrow Q \times I$ be given by the known homotopy from $f$ to $g$. Now $q+1 \geqq(n+1)+3, M \times I$ is $2(n+1)-(q+1)$ connected and $Q \times I$ is $2(n+1)-(q+1)$ +1 connected. By Theorem $1, F \sim H$ rel $M \times I$ where $H$ is a proper locally flat imbedding. 


\section{REFERENCES}

1. M. Brown, Locally flat imbeddings of topological manifolds, Ann. of Math. (2) 75 (1962), 331-341. MR 24 \#A3637.

2. J. Dancis, Approximations and isotopies in the trivial range, Topology Seminar (Wisconsin, 1965), Ann. of Math. Studies, no. 60, Princeton Univ. Press, Princeton, N. J., 1966, pp. 171-187. MR 36 \#7144.

3. - Some nice embeddings in the trivial range, Topology Seminar (Wisconsin, 1965), Ann. of Math. Studies, no. 60, Princeton Univ. Press, Princeton, N. J., 1966, pp. 159-170. MR 36 \#3331.

4. A. Haefliger, Plongements différentiables de variétés dans variétés, Comment. Math. Helv. 36 (1961), 47-82. MR 26 \#3069.

5. S. Hu, Homotopy theory, Pure and Appl. Math., vol. 8, Academic Press, New York, 1959. MR 21 \#5186.

6. J. F. P. Hudson, Piecewise linear topology. Vol. I, The University of Chicago, Chicago, Ill., 1967 (mimeographed).

7. M. C. Irwin, Embeddings of polyhedral manifolds, Ann. of Math. (2) 82 (1965), 1-14. MR 32 \#460.

8. R. C. Kirby and L. C. Siebenmann, For manifolds the Hauptvermutung and the triangulation conjecture are false, Notices Amer. Math. Soc. 16 (1969), 695. Abstract \#69T-G80.

9. J. A. Lees, An engulfing theorem for topological manifolds, Rice University, Houston, Tex., 1968 (mimeographed).

10. — Locally flat embeddings of topological manifolds, Ann. of Math. (2) 89 (1969), 1-13. MR 38 \#6563.

11. R. T. Miller, Close isotopies on piecewise linear manifolds, Trans. Amer. Math. Soc. 151 (1970), 597-628.

12. J. R. Munkres, Elementary differential topology, rev. ed., Ann. of Math. Studies, no. 54, Princeton Univ. Press, Princeton, N. J., 1966. MR 33 \#6637.

13. M. H. A. Newman, The engulfing theorem for topological manifolds, Ann. of Math. (2) 84 (1966), 555-571. MR 34 \#3557.

14. E. H. Spanier, Algebraic topology, McGraw-Hill, New York, 1966. MR 35 \#1007.

15. R. G. Swan, The theory of sheaves, Univ. of Chicago Press, Chicago, Ill., 1964.

16. G. P. Weller, Locally flat imbeddings of topological manifolds, Ph.D. Thesis, University of Chicago, Chicago, Ill., 1968.

17. E. C. Zeeman, Unknotting combinatorial balls, Ann. of Math. (2) 78 (1963), 501-526. MR 28 \#3432.

\section{University of Illinois at Chicago Circle,} Chicago, Illinois 60680 